\title{
Program Design of JSON to Structured Data Conversion
}

\author{
Zhongyue Da ${ }^{1, a}$, Wenjie Yang ${ }^{l}$, Peipei Ran ${ }^{1}$, Yuke Huo ${ }^{l}$ \\ ${ }^{1}$ School of Printing \& Packaging Engineering, Beijing Institute of Graphic Communication, Beijing 102600, PR China
}

\begin{abstract}
With the maturity of structured data storage technology and the increasing demand for enterprise information development, some enterprises need to convert the data storage model of application system from JSON to structured data. A file management system is token as an example to explain how to convert. In this article, it is achieved through the following three parts. First, the structure diagram of data transformation is built. Then, JSON model and structured data model are built. Finally, the conversion of data from JSON to MySQL is realized by using java language.
\end{abstract}

\section{Introduction}

With the development of relational database technology, relational database systems are becoming more stable and efficient. Relational data are studied to store semi-structured data in many enterprises, whose goal is to share data between multiple different system environments [1]. And it is beneficial to the diversified development and progress of the application system.

In non-database environments, semi structured data is common, such as XML and JSON [2]. The latter is discussed in this paper. JSON is easy to read and write, and is easily parsed and produced by machines. JSON format is not only language independent text format, but also conforms to the habit of C language family [3].

In the actual application of enterprises, different data storage technologies are used in different network systems. For example, in a file management system, two sets of storage methods are used, JSON semi-structured storage and MySQL database storage. To facilitate the application and rapid query, it is necessary to convert and merge the data storage mode. In this paper, the file management system is taken as an example to discuss and implement the conversion from JSON to relational database.

\section{Program Design}

\subsection{Demand analysis}

In this paper, the data conversion in a file management system is taken as an example to illustrate the process, which needs to get date from JSON file and store it into MySQL database. JSON file is composed of JSON [4] and unstructured binary data. Before storing the JSON data, the original files should be pre-processed to achieve the purpose of splitting binary data and JSON data. After it, semi-structured data is converted into JSON documents, while binary data is stored as pictures.

\subsection{The overall design}

The whole process of data conversion includes pre-processing, mode analysis, mode conversion, data conversion, data storage and so on. The specific process is shown in figure 1.

a Corresponding author: dazhongyue@qq.com 


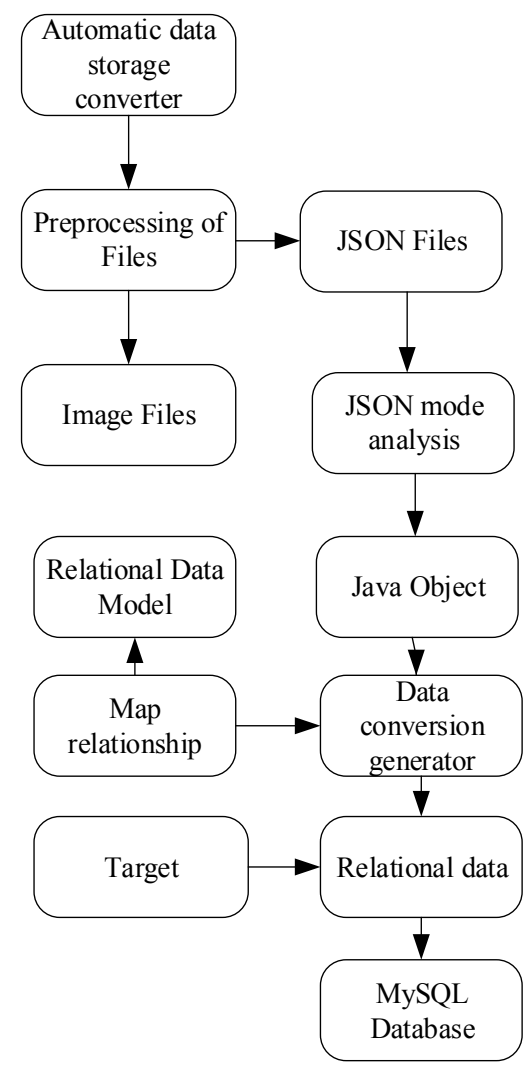

Fig. 1. Schematic diagram of the structure

\section{Model Analysis}

Schema transformation is the foundation of the whole process. The task needs to be accomplished is to build JSON semi-structured data model and generate corresponding relational data table model, then process JSON semi-structured data into structured data and store it in database.

\subsection{Model Analysis}

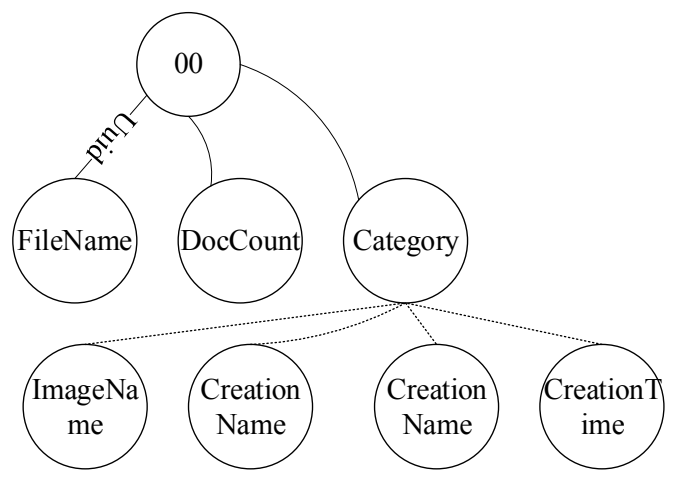

Fig.2. JSON Model

As shown in Figure two, the JSON data is deeply parsed, and then the structure model diagram is built, which is a simplified JSON OEM model diagram [5].
First, the types of nodes are partitioned, the node Category of the pattern division is found. Because Its child nodes are only one layer and the number of child nodes is appropriate. Other nodes are considered as data nodes.

Second, based on the previous results, two tables, info and file, were created. All the data nodes are put in the nodes of the model they belong to. According to the actual situation, attributes are added to type.

Third, according to the subordination in the OEM model [5], a master-slave table relation is generated, and a foreign key index is established between the two tables. Info is the primary table, and File is a child table. The foreign key Mainno in the table corresponds to FileName in JSON.

After the above process, the JSON tree structure in Figure 2 is converted to the table structure of Table 1 and Table 2.

Table 1. Information table(info)

\begin{tabular}{|l|l|l|}
\hline Properties & Data type & Meaning \\
\hline Mainno & VARCHAR & Primary key \\
\hline Count & INT & File number \\
\hline CreateName & VARCHAR & Record man \\
\hline CreateTime & DATETIME & Record time \\
\hline
\end{tabular}

Table 2. File storage table (file)

\begin{tabular}{|l|l|l|}
\hline Properties & Data type & Meaning \\
\hline ID & INT & Primary key \\
\hline Mainno & VARCHAR & Foreign key \\
\hline imageName & VARCHAR & File name \\
\hline dir & VARCHAR & File directory \\
\hline remark & VARCHAR & Remark \\
\hline
\end{tabular}

\subsection{Model implementation}

The JSON document storage and MySQL database storage model is described in figure 3. The following is a description of the data model:

In the JSON document storage model, FileName is the name of the document, RecordDate is the document creation date, count is the number of images that the document contains, and imageName is the name of the picture. Remark is a note.

The attributes of the table in the chart are shown in Table 1 and Table 2. 


\begin{tabular}{|c|c|}
\hline JSON & Classes \\
\hline $\begin{array}{l}\text { "FileName": "', } \\
\text { "Expected": "', } \\
\text { "DocCount": , } \\
\text { "Category": \{ } \\
\text { "ImageName": "', } \\
\text { "CreationName": "', } \\
\text { "CreationName": "'" } \\
\text { \}, } \\
\text { "Remark": null }\end{array}$ & $\begin{array}{l}\text { class file }\{ \\
\text { String FileName; } \\
\text { String Expected; } \\
\text { String DocCount; } \\
\text { Category category; } \\
\text { class Category \{ } \\
\text { String ImageName; } \\
\text { String CreationName; } \\
\text { String CreationName; } \\
\text { String Remark;\} }\end{array}$ \\
\hline Tables & Classes \\
\hline $\begin{array}{l}\text { Table info } \\
\text { Cloumn Mainno } \\
\text { Cloumn Count } \\
\text { Cloumn CreationTime } \\
\text { Cloumn CreationName } \\
\text { Table File } \\
\text { Cloumn mainno } \\
\text { Cloumn ImageName } \\
\text { Cloumn dir } \\
\text { Cloumn remark }\end{array}$ & $\begin{array}{l}\text { class info \{ } \\
\text { String Mainno; } \\
\text { String Count; } \\
\text { Date CreationTime; } \\
\text { String CreationName; } \\
\text { class File }\{ \\
\text { String mainno; } \\
\text { String ImageName; } \\
\text { String dir; } \\
\text { String remark; }\}\end{array}$ \\
\hline
\end{tabular}

Fig.3 JSON model transformation diagram

\section{The implementation of conversion}

\subsection{Data analysis}

When the schema transformation is completed, the application data can be transformed into the target mode according to its corresponding relationship, and the basic process is divided into 3 steps:

(1) Read the JSON document data, get JSON format data;

(2) Translate from the original data to the target model of data according to the corresponding relationship between the data in Figure 2;

(3) Write the second step of the data to the MySQL database.

\subsection{Program implementation}

The following are classes implemented by the conversion program, as shown in figure 4.

(1) Doc class represents a data model class of JSON, and other data models are not described

(2) UpdateAction class belongs to the database connection class

(3) JSONAysis and AysisRead class, belonging to the JSON object read and model conversion class
(4) Read class is the core class, used to call other classes and complete all the steps of a file.

(5) The Account class is a target model class, where setter and getter represent the get and set functions for all attributes. Other data models are not described.

(6) FileOut class is the picture file output class, which converts binary data into JPG images.

\subsection{Matters need attention}

In the process of technical implementation, we should pay attention to the following aspects.

(1) In the aspect of time type transformation, the time expression format in JSON is dependent on the environment because JSON does not contain a variable type. First, the program reads data in a string, then determines the type, converts the format, and stores it in the database. If not, the system can't work. For example, "2017-03-27T16:10:11.626398+08:00", "2017-03-27", "2017-03-27T16:10:11.626+08:00" and other expressions are translated into "2017-03-27"".

(2) When reading multiple byte numbers (such as 16-bit integers), we should pay attention to the problems of high byte and low byte order. For example, when IO is written to a file, the order of bytes written by the $\mathrm{C}++$ is from low to high, while Java is from high to low.

(3) The following code is added to avoid the running error when the JSON is serialized. Because the JSON object and the JSON structure are not one-to-one.

objectMapper.configure(DeserializationFeature.F AIL_ON_UNKNOWN_PROPERTIES, false);

\section{Conclusions}

In this paper, it discusses process from design model to the source code and gradually studies the storage of semi-structured data. First, the flow chart is used to describe the whole process of program implementation. Then the storage model of semi-structure data is constructed by using OEM model [5]. Next, according to the mapping rules, the JSON storage model is mapped to structed data, and 
data conversion rules from JSON to MySQL are implemented. In addition, the program has been successfully applied to the file management system, which has realized the data conversion and achieves good results. The next step is to achieve dynamic transformation between semi-structured data and structured data

Acknowledgments: The study is supported by the 'Development and application of micron level vision quality detector (2013YQ140517)'.

\section{References}

1. Wang Wei. Zhu Dong. Peng Kaiyuan. From file system to database system: automatic conversion method and practice of application software. The Tenth National Academic Conference on Database, 608-612
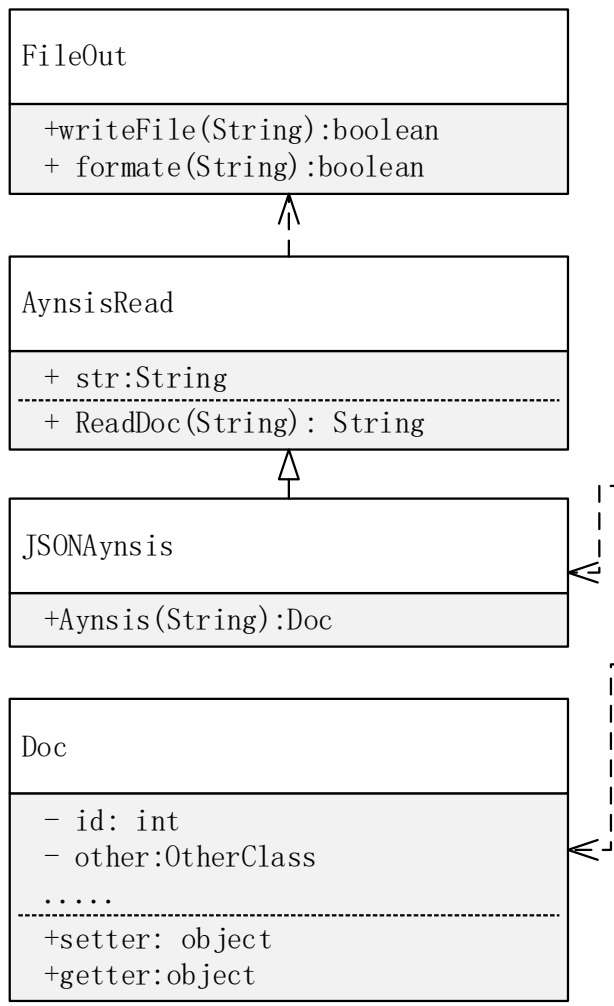

Fig. 4. Class Diagram of Program Implementation
2. Xiaoyue Xin. Design and Research Study on Data Model of Document Database. Sun Yat-sen University (2015).

3. Fellipe Freire, Crishane Freire and Damires Souza Academic Unit of Informatics, Federal Institute of Education, Science and Technology of Paraiba, João Pessoa, Brazil Enhancing JSON to RDF Data Conversion with Entity Type Recognition (2017) . 4. Huyin Zhang, Qiansong Qu, Ruiyun Hu. Data exchange model based on JSON, Computer engineering and design.36(2015), 3380-3384 5. HangYujia1, SuZhongbin, WuHuarui. ZhuHuaji. TaoYong. RESEARCH ON DYNAMIC TREE STORAGE MODEL OFSEMI-STRUCTURED

DATA, Computer Applications and Software, 28, (2011), 86-90.

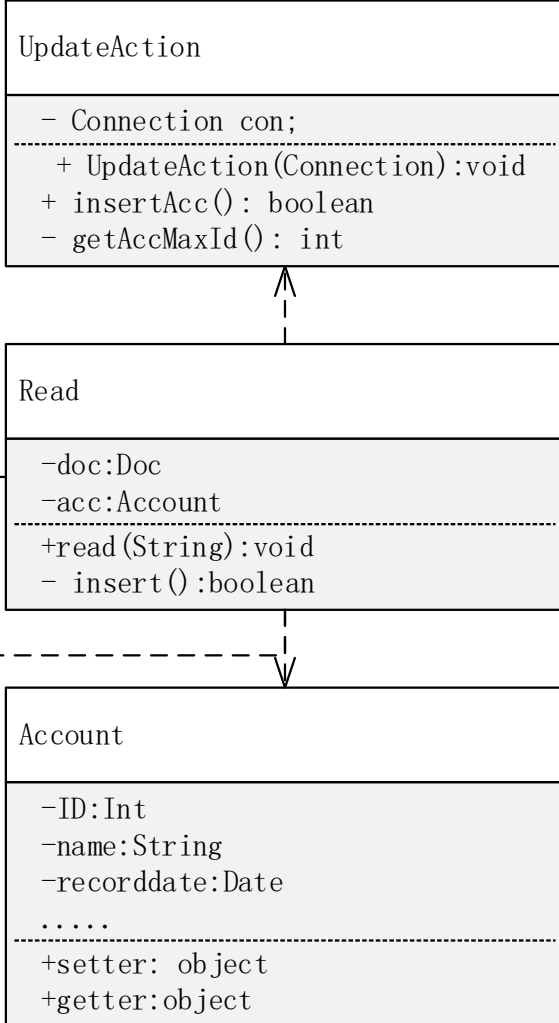

